\title{
Diagnosing intramammary infections: Comparison of multiple versus single quarter milk samples for the identification of intramammary infections in lactating dairy cows
}

\author{
I. Dohoo, ${ }^{\star 1}$ S. Andersen, ${ }^{*}$ R. Dingwell,† K. Hand,‡ D. Kelton,§ K. Leslie,§ Y. Schukken,\# and S. Goddenll \\ ${ }^{*}$ Centre for Veterinary Epidemiological Research, University of Prince Edward Island, 550 University Avenue, Charlottetown, PE, C1A 4P3, \\ Canada \\ †Holdrege Vet Clinic PC, 2201 4th Avenue, Holdrege, NE 68949 \\ ¥Strategic Solutions Group, 142 Hume Rd. RR1, Puslinch, ON, N0B 2J0, Canada \\ $\S$ Department of Population Medicine, University of Guelph, Guelph, ON, N1G 2W1, Canada \\ \#Department of Population Medicine and Diagnostic Sciences, and Clinical Sciences, Cornell University, Ithaca, NY 14853 \\ IIDepartment of Veterinary Population Medicine, College of Veterinary Medicine, University of Minnesota, Saint Paul 55108
}

\section{ABSTRACT}

The objective was to examine the potential benefits of using different combinations of multiple quarter milk samples compared with a single sample for diagnosing intramammary infections (IMI) in dairy cattle. Data used in the analyses were derived from 7,076 samples from 667 quarters in 176 cows in 8 herds in 4 locations (Minnesota/Wisconsin, $\mathrm{n}=4$; Prince Edward Island, $\mathrm{n}=2$; Ontario, $\mathrm{n}=1$; New York, $\mathrm{n}=1$ ). Duplicate quarter milk samples were collected at morning milking for 5 consecutive days. Cows were evenly distributed between early postparturient and mid- to late-lactation cows. All samples were frozen for shipping and storage, thawed once, and cultured in university laboratories using standardized procedures consistent with National Mastitis Council guidelines. The presence of specific pathogens was confirmed and identified using the API identification system (bioMerieux, Marcy l'Etoile, France) in each laboratory. A previously developed gold standard was applied to the first sample from d 1, 3, and 5 to classify infected quarters. The data were analyzed separately for coagulase-negative staphylococci (CNS) and Streptococcus spp. Various combinations of test results from d 2 and 4 were used in the test evaluation. These consisted of single samples $(n=4), 2$ sets of duplicate samples (2 samples collected on the same day), 2 sets of consecutive samples (2 samples collected $2 \mathrm{~d}$ apart), and 2 sets of triplicate samples (2 samples on the same day and a third sample $2 \mathrm{~d}$ apart). Series interpretation of duplicate or consecutive samples (i.e., positive $=$ same pathogen isolated from both samples) resulted in the highest specificity (Sp; CNS Sp $=92.1-$ 98.1\%; Streptococcus spp. Sp = 98.7-99.6\%), but lowest

Received April 26, 2011.

Accepted July 6, 2011.

${ }^{1}$ Corresponding author: dohoo@upei.ca sensitivity (Se; CNS Se $=41.9-53.3 \%$; Streptococcus spp. Se $=7.7-22.2 \%)$. Parallel interpretation of duplicate or consecutive samples (i.e., positive $=$ pathogen isolated from either) resulted in the highest Se (CNS $\mathrm{Se}=70.8-80.6 \%$; Streptococcus $\mathrm{spp} . \mathrm{Se}=31.6-48.1 \%$ ), but lowest Sp (CNS Sp $=72.0-77.3 \%$; Streptococcus spp. Sp $=89.5-93.3 \%)$. The difference in estimates between single and duplicate samples was larger than between single and consecutive samples. Overall, triplicate samples provided the best combination of Se and Sp, but compared with a single sample, provided only a modest gain in Sp and little or no gain in Se.

Key words: intramammary infection, triplicate, sensitivity, specificity

\section{INTRODUCTION}

Worldwide, mastitis is one of the most economically important diseases in dairy production, with subclinical mastitis accounting for almost two-thirds of economic loss (Seegers et al., 2003; Halasa et al., 2007). Subclinical mastitis implies inflammation within the udder but not necessarily infection. The inflammatory reaction may be identified by an elevated SCC or other measure of inflammation (e.g., California Mastitis Test). Subclinical mastitis is most often due to a bacterial IMI (Djabri et al., 2002), so the terms IMI and subclinical mastitis are often used interchangeably (Barkema et al., 1997; Deluyker et al., 2005). The term IMI refers specifically to the presence of an infectious organism in the udder (Berry and Meaney, 2006). Because of the central role of IMI in mastitis, high-quality information is required on the operating characteristics [sensitivity (Se) and specificity $(\mathbf{S p})$ ] of procedures used to classify mammary glands as having an IMI or not for correct interpretation of test results.

Historically, several definitions of mastitis and IMI have been used. In 1987, both the International Dairy 
Federation (1987) and the National Mastitis Council (1987) published definitions and guidelines for diagnosis of bovine mastitis and IMI. More recently, the Se and $\mathrm{Sp}$ of a variety of definitions for an IMI have been published (Dohoo et al., 2011).

Both the International Dairy Federation and the National Mastitis Council (NMC) recommended taking multiple samples from individual mammary glands to positively identify IMI (International Dairy Federation, 1987; National Mastitis Council, 1999, 2004). This view was presented in an FDA Guidance Document on evaluation of drug safety and effectiveness for antimicrobial bovine mastitis products (US FDA, 1996). The guidelines described the use of duplicate milk samples, interpreted in series, to establish IMI status before and after testing the drug. It was suggested that use of a single sample might be an acceptable practice for contagious pathogens, but not for environmental pathogens (Erskine and Eberhart, 1988). In general, objective information about the relative merits of using duplicate or triplicate samples compared with single samples is lacking. Because bacteriological culture is frequently the most expensive component in an experimental trial, use of duplicate or triplicate samples may adversely affect a study by limiting the sample size of the trial.

This study was conducted as one component in a multi-part process with the overall goal of determining the operating characteristics of various definitions of IMI. The first step was to develop a consensus gold standard based on 3 consecutive milk samples (Andersen et al., 2010). Recent manuscripts have addressed the issue of diagnosing an IMI based on a single milk sample (Dohoo et al., 2011) and evaluated the use of composite milk samples (Reyher and Dohoo, 2011).

The objective was to examine the potential benefit of using different combinations of multiple mammary gland milk samples compared with single samples for diagnosing IMI. The combinations of single, duplicate, consecutive, and triplicate milk samples were compared with the consensus gold standard established by Andersen et al. (2010).

\section{MATERIALS AND METHODS}

\section{The Data}

The data were collected during summer 2003 from 9 herds at 4 different locations: US Midwest - Minnesota/ Wisconsin $(\mathrm{n}=5)$; eastern Canada - Prince Edward Island (PEI; $\mathrm{n}=2)$; central Canada - Ontario ( $\mathrm{n}=$ $1)$; northeastern US - New York $(\mathrm{n}=1)$. Duplicate quarter milk samples (referred to as A and B samples) were collected at morning milking for 5 consecutive days from a total of 197 cows, evenly distributed among fresh and mid- to late-lactation cows. Fresh cows were defined as being close to calving ( 1 to $5 \mathrm{~d}$ in milk if possible). Mid- to late-lactation cows were defined as being at least $150 \mathrm{~d}$ in milk at $\mathrm{d} 1$ of sampling. The cows were randomly selected among those in the herd with no recent or concurrent clinical mastitis. Each cow's parity and days in milk at the start of the sampling was recorded. Samples from d 1, 3, and 5 made up the triplicate set that was used to classify the quarter as having, or not having, an IMI with a specific pathogen for the 5 -d period. Samples from d 2 and $4(\mathrm{n}=4)$ were evaluated individually for their ability to correctly classify the quarter.

All samples were frozen for storage, thawed once and a $0.01-\mathrm{mL}$ sample cultured in university laboratories using standardized procedures consistent with NMC guidelines. Samples from the Canadian sites were cultured at the University of Guelph and samples from the US sites were cultured at the University of Minnesota. The presence of specific pathogens was confirmed and identified using the API identification system from BioMérieux (Marcy l'Etoile, France) in each laboratory. Isolates were classified into 1 of 21 different species or groups of species, although only 2 categories, CNS and Streptococcus spp. (consisting primarily of Strep. uberis with a moderate number of Strep. dysgalactiae and very few Strep. agalactiae and Strep. bovis), had sufficient numbers of isolates to be used in this study.

\section{Gold Standard Definition}

The gold standard definition used was based on the published consensus standard definition (Andersen et al., 2010). A sample was considered gold standard-positive (GS+) for a given pathogen if the same pathogen was isolated from 2 of 3 samples collected on $\mathrm{d} 1,3$, and 5 or if the d 3 sample was culture-positive with more than 10 colonies $(1,000 \mathrm{cfu} / \mathrm{mL}$ of milk) isolated. In all cases, the A sample from each day was used in the evaluation unless it was contaminated, in which case the B sample was used. Samples that were not GS+ were considered gold standard-negative (GS-).

\section{Sample Combinations Evaluated}

Sample combinations evaluated used samples collected on d 2 and 4 and consisted of

- single samples (for comparison purposes): all 4 single samples (A and B on d 2 and 4) were considered $(\mathrm{n}=2,415)$ 
- duplicate samples (2 samples collected at the same milking): both the d 2 pair and the d 4 pair were evaluated $(\mathrm{n}=1,187)$

- consecutive samples (a pair of samples collected on d 2 and 4): both the A and B sample pairs were evaluated $(\mathrm{n}=1,089)$

- triplicate samples $(2$ samples collected on $1 \mathrm{~d}$ and a third on the other day; i.e., 2 samples from d 2 with the third from d 4 , or 2 from d 4 with the third from $\mathrm{d} 2 ; \mathrm{n}=1,068)$. Note: in practice, the third sample would need to be collected only if the first 2 were in disagreement, but in this study all samples had been collected and stored.

For both duplicate and consecutive samples, results were interpreted both in series (positive $=$ same pathogen isolated from both samples) and in parallel (positive $=$ pathogen isolated from either). For the triplicate samples, the sample was considered positive if 2 of the 3 samples yielded the same pathogen. Each sample was considered positive for a pathogen if the pathogen was isolated, regardless of the number of colonies found (minimum colony count was $100 \mathrm{cfu} / \mathrm{mL}$ ).

\section{Analyses}

Descriptive Statistics and Unconditional Associations. For each species group, descriptive statistics, including the number of quarters that were classified GS+ and GS - were computed. For each combination of samples and each species of interest, the Se and Sp were computed (Dohoo et al., 2009). The confidence interval around these estimates was determined using formulae appropriate for binomial proportions. Plots of Se and Sp and their 95\% confidence interval (CI) from unconditional analyses were created for each species combination.

Random Effects Models. Because observations (samples) were not independent, but rather were clustered within mammary glands (multiple observations per individual mammary glands), within cows (4 glands per cow), and within herds, the CI estimated above were likely underestimates of the true CI for the Se and Sp. Consequently, random effects logistic regression analyses were carried out with random effects for herd, cow, and mammary gland. Maximum likelihood estimation of these models was performed using adaptive quadrature methods. Separate analyses were carried out for GS+ and GS - sets of samples, with the test result being the outcome of interest. Estimates derived from these models were converted to marginal (or population average) estimates based on the total variance of the random effects (Dohoo et al., 2009). Plots comparing selected estimates of Se and Sp and
Table 1. Distribution of number of organisms isolated from each milk sample with valid culture results for identification of IMI

\begin{tabular}{lrc}
\hline $\begin{array}{l}\text { Species } \\
\text { isolated (no.) }\end{array}$ & $\begin{array}{c}\text { Samples } \\
\text { (no.) }\end{array}$ & $\begin{array}{c}\% \text { of } \\
\text { total }\end{array}$ \\
\hline Negative & 4,355 & 61.6 \\
1 & 2,086 & 29.5 \\
2 & 609 & 8.6 \\
3 & 26 & 0.4 \\
Total & 7,076 & 100 \\
\hline
\end{tabular}

their 95\% CI from unconditional analyses and random effects models were created for CNS. All analyses were carried out using Stata (version 11, StataCorp, 2009).

\section{RESULTS}

\section{Descriptive Statistics}

Data were recorded on 7,630 samples, of which 7,076 provided usable culture results [554 samples (7.3\%) contaminated or data missing]. The distribution of number of organisms isolated from each sample is given in Table 1. Only 26 samples yielded 3 different organisms.

In total, 667 individual mammary glands from 176 cows in 8 herds had sufficiently complete bacteriology to enable the GS status of the gland to be determined (based on samples from d 1,3, and 5) and one or more valid samples from $\mathrm{d} 2$ and 4 . Of these individual glands, 211 were classified as GS+ for CNS, 39 for Streptococcus spp., and 42 for enterococci. This last group of organisms was considered of less interest and results were broadly similar to those obtained from CNS and Streptococcus spp., so enterococci will not be discussed further. For all other pathogens of interest, 5 or fewer individual glands were GS+ and had insufficient data for analysis.

\section{Unconditional Estimates of Se and Sp}

Unconditional estimates (and CI) of Se and Sp were obtained for each sample combination considered. The results are presented in Figure 1 (CNS) and Figure 2 (Streptococcus spp.). Summary estimates of Se and Sp for each sample combination (averaged across the pair of results) are given in Table 2. Use of a single sample resulted in low Se (61.2\%) and moderate Sp (84.3\%) for CNS (Table 2). For Streptococcus spp., the Se was very low $(29.0 \%)$ and Sp was high $(94.8 \%)$.

As expected, series interpretation (both samples had to be positive for the individual gland to be positive) resulted in lower Se and higher Sp than a single sample. For both organism groups (CNS and Streptococcus spp.), the difference between the single sample estimate 


\section{CNS}
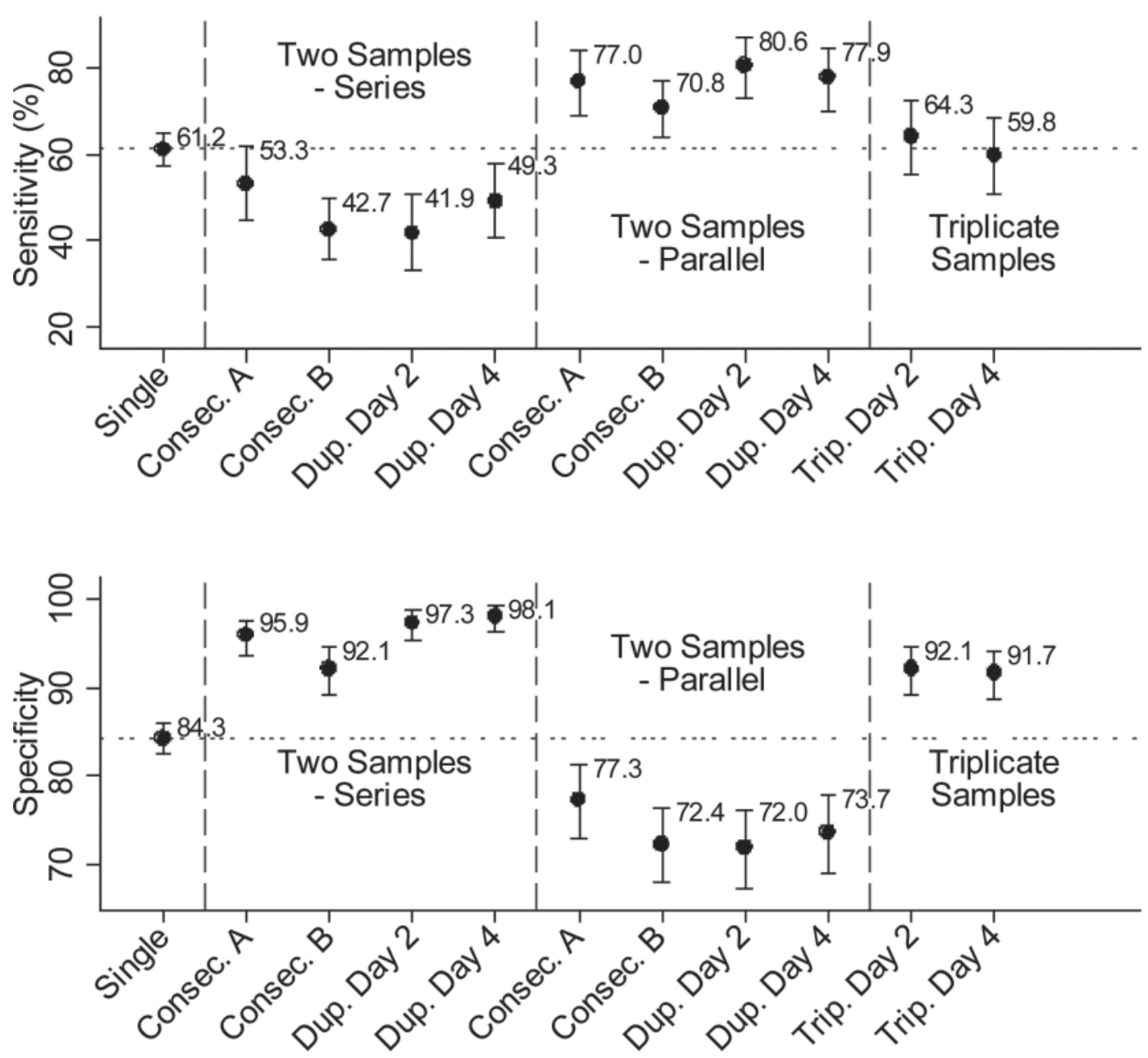

Figure 1. Estimates (and 95\% CI) of sensitivity and specificity for different sampling combinations for the diagnosis of an IMI with CNS. Two samples (designated A and B) were collected on each of d 2 and 4. Single samples were either the A or B sample from d 2 or 4 (estimates pooled). Consecutive samples were the A set or B set samples taken on d 2 and 4 . Duplicate samples were the pair (A and B) of samples collected on the same day (2 or 4). Triplicate samples had 2 samples collected on either d 2 or 4 with the 3rd sample from the other day.

and the average of the duplicate sample estimates was larger than that between the single sample estimate and the average of the consecutive sample estimates. This was true for both Se and Sp. On the other hand, parallel interpretation (either sample being positive resulted in the quarter being considered positive) resulted in higher Se, but lower Sp than a single sample did. Once again, for both Se and Sp, the average of the duplicate sample estimates was generally further from the single sample estimate than was the average of the consecutive sample estimates (Sp for Streptococcus spp. being the exception).

Triplicate sampling resulted in little or no improvement in Se and only a moderate improvement in Sp compared with use of a single sample. For CNS, the
2 Sp estimates were 92.1 and $91.7 \%$ for triplicates compared with $84.3 \%$ for a single sample (Figure 1). For Streptococcus spp., the gain in Sp was even smaller (98.2 and 97.6 vs. 94.8; Figure 2), possibly because of the higher level of Sp for a single sample (i.e., less room for improvement).

\section{Random Effects Models}

A comparison of unconditional and random effects estimates of Se and Sp for CNS for a subset of the sample combinations is in Figure 3. These included single samples (all 4 samples), duplicate samples from d 2 interpreted in both series and parallel, and triplicate samples (2 samples from d 4, with a d 2 sample as the 
Table 2. Mean sensitivities and specificities for each of the sampling combinations (averaged over the 2 sets of observations for each sampling combination) for identification of IMI

\begin{tabular}{lccccc}
\hline & \multicolumn{2}{c}{ CNS } & & \multicolumn{2}{c}{ Streptococcus spp. } \\
\cline { 2 - 3 } \cline { 5 - 6 } Sampling method $^{1}$ & Sensitivity & Specificity & & Sensitivity & Specificity \\
\hline Single sample & 61.2 & 84.3 & & 29.0 & 94.8 \\
Consecutive (series) & 48.0 & 94.0 & & 20.3 & 98.8 \\
Duplicate (series) & 45.6 & 97.7 & & 9.4 & 99.4 \\
Consecutive (parallel) & 73.9 & 74.9 & & 38.0 & 91.4 \\
Duplicate (parallel) & 79.3 & 72.9 & & 47.2 & 91.5 \\
Triplicate & 62.1 & 91.9 & & 22.5 & 97.9 \\
\hline
\end{tabular}

${ }^{1}$ Consecutive samples were 2 samples collected $2 \mathrm{~d}$ apart; duplicate samples were 2 samples collected at the same milking; triplicate samples were 2 samples from $1 \mathrm{~d}$ with the third $2 \mathrm{~d}$ later (or earlier). Series interpretation meant that the result was considered positive if either of the 2 samples was positive, whereas parallel interpretation required both samples to be positive.

Strep. spp.
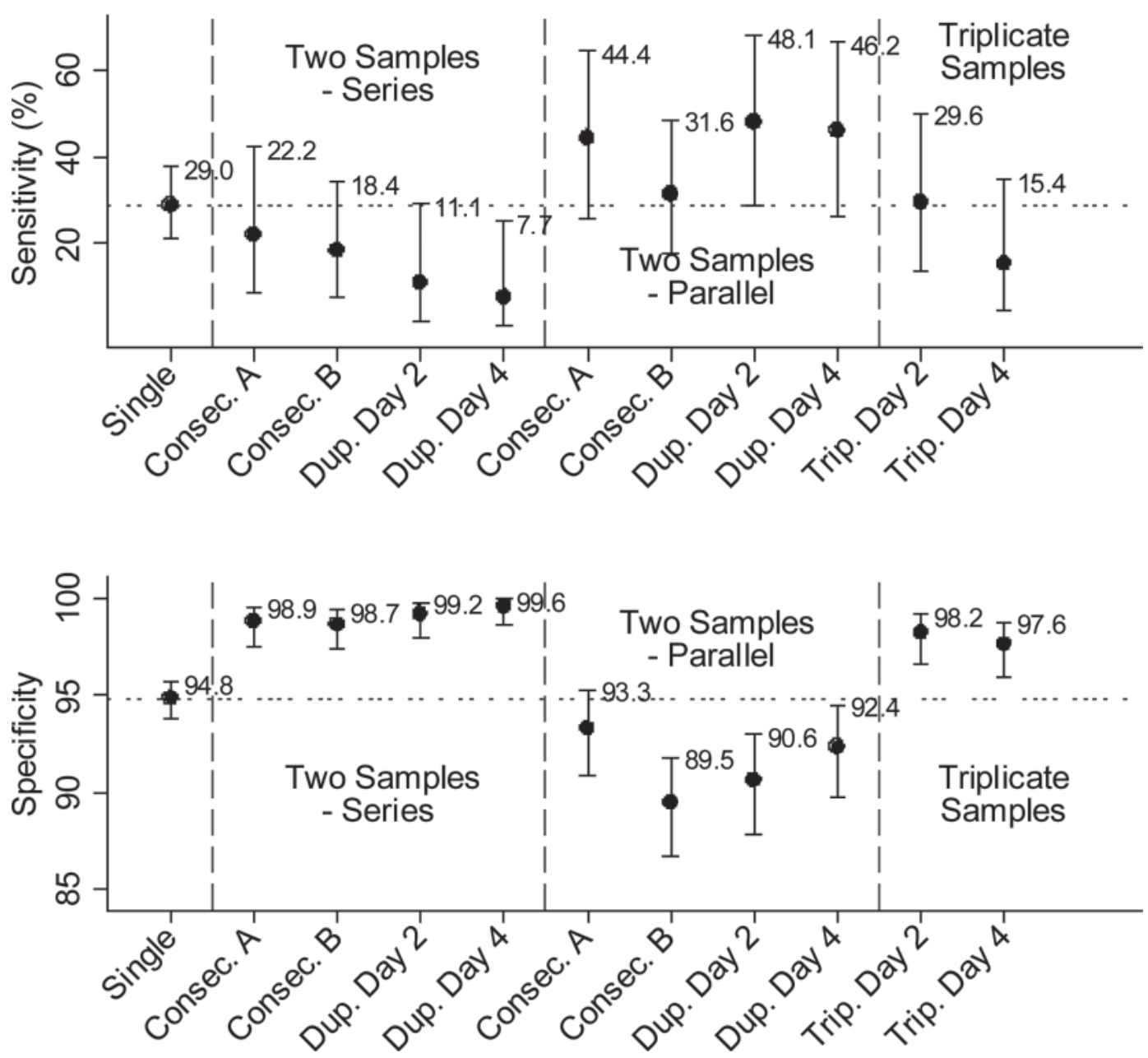

Figure 2. Estimates (and 95\% CI) of sensitivity and specificity for different sampling combinations for the diagnosis of an IMI with Streptococcus spp. Two samples (designated A and B) were collected on each of d 2 and 4. Single samples were either the A or B sample from d 2 or 4 (estimates pooled). Consecutive samples were the A set or B set samples taken on d 2 and 4 . Duplicate samples were the pair (A and B) of samples collected on the same day (2 or 4). Triplicate samples had 2 samples collected on either d 2 or 4 with the third sample from the other day. 


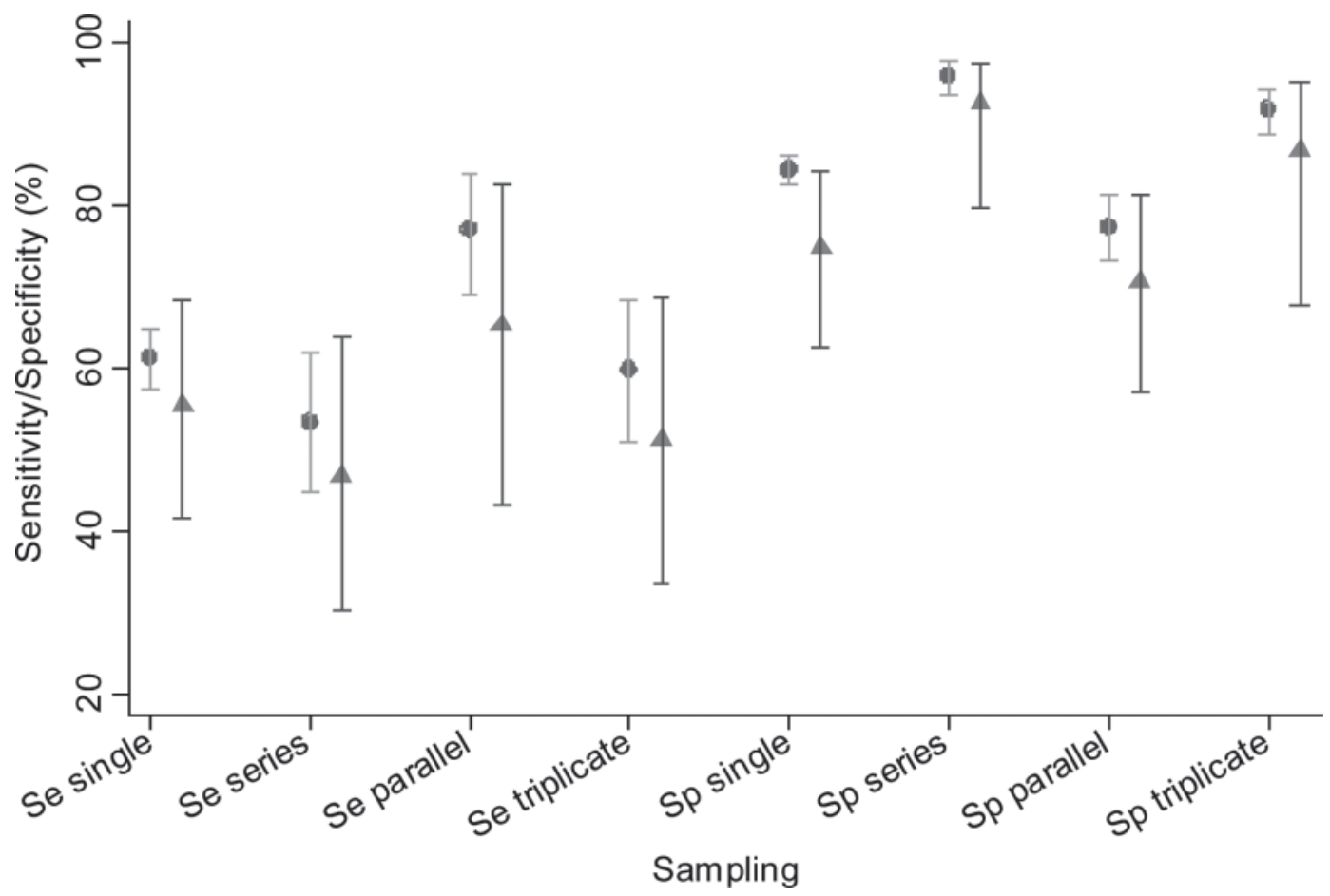

Figure 3. Comparison of unconditional estimates (circle) of sensitivity (Se) and specificity (Sp) for diagnosing an IMI with CNS to those derived from random effects models (triangle). Single sample results were pooled across all 4 single samples evaluated (d 2 and 4 , samples A and B). Consequently, the confidence interval (CI) for single samples is much narrower than that for other sample combinations, which are presented individually.

third sample). All estimates from the random effects models were lower than those from the unconditional analyses and they had much wider CI. Comparable results were observed for other sample combinations and for Streptococcus spp. (data not shown).

\section{DISCUSSION}

\section{Study Populations}

Because intensive sampling was required for this project, herds were purposively selected. They were broadly based geographically (New York, Ontario, Prince Edward Island, and Minnesota/Wisconsin) and considered representative of the dairy populations in those regions. The data required to estimate the operating characteristics (Se and $\mathrm{Sp}$ ) of a test are extensive. Even though over 7,500 milk samples were cultured, there were only enough individual mammary glands considered GS+ from 2 important groups of species included in the analyses. The effects of different sampling combinations on Se and Sp were similar for CNS and Streptococcus spp., so it is likely that similar effects would be observed for other pathogen groups.

\section{Gold Standard}

In the absence of a perfect gold standard, using the best diagnostic protocol available as the gold standard is important in any test evaluation. Using culture results from triplicate quarter milk samples has often been considered the gold standard for classifying individual mammary glands with regard to IMI status. It was recently reported that, even when complete data from 3 independent samples are available, considerable disagreement can exist among mastitis experts as to the probability of an IMI within the quarter (Andersen et al., 2010). Consequently, a consensus standard agreed to by participants in the Mastitis Research Workers' Conference was used as the gold standard for this evaluation.

Consideration must be given to what biases may have resulted from the use of an imperfect gold standard. Truly uninfected quarters were unlikely to meet the criteria laid out to be considered GS+, so there were probably very few false positives among the GS+ samples. Some quarters with an IMI may have failed to meet the criteria required to be considered GS+. There are 2 consequences of this misclassification. First, quarters classified as GS- would have included some truly infected quarters. If these came up positive on one or more of the sample combinations, they would have been incorrectly classified as false positives. Consequently, the estimates of $\mathrm{Sp}$ in this paper may be somewhat negatively biased. However, given that nearly all the Sp estimates were very high, the magnitude of this 
bias cannot have been large. The second consequence of missing some infected quarters from the GS+ group was that quarters classified as GS+ represented a biased subset of all infected quarters, and samples in this subset were presumably easier to classify as positive based on the various sample combinations. Consequently, the estimates of Se derived in this paper may well be biased upward. However, because the potential bias described above would have applied to all sample combinations, differences in Se or Sp between sample combinations were not expected to have a bias.

\section{Unconditional Estimates of Se and Sp}

Unconditional estimates of Se and Sp from single samples were in agreement with those previously published (Dohoo et al., 2011). Duplicate sampling with the results interpreted in series is frequently used in mastitis research and has been considered a gold standard (National Mastitis Council, 2004; Torres et al., 2009). Duplicate samples have an overall high level of agreement (Jasper et al., 1974), but lower levels of agreement were reported for Streptococcus spp. and coliform organisms (Erskine and Eberhart, 1988). The results from this study confirm that this combination results in high $\mathrm{Sp}$, but the low Se of single sample procedures is made substantially worse. The increase in Sp and decrease in Se was more extreme for duplicate samples than for consecutive samples. This is not surprising because it has been shown that duplicate samples have a higher level of agreement than do consecutive samples (Postle, 1976).

Duplicate sampling with results interpreted in parallel is used much less frequently than series interpretation. The results of this study show that this combination should be considered in situations in which a high, or at a least moderate, level of Se is required. For example, in studies on the elimination of infections (e.g., over the dry period), one needs to be certain that an infection has been eliminated, so using a sensitive procedure for detecting infections at the end of the study period would be warranted.

Triplicate sampling was considered a gold standard for detection of IMI (Torres et al., 2009); indeed, the gold standard classification of individual glands in this study was based on triplicate samples. It is clearly not a perfect gold standard, or we would have observed perfect agreement $(\mathrm{Se}=\mathrm{Sp}=100 \%)$ between our gold standard classifications and the triplicate test results. Although triplicate sampling did achieve relatively high levels of Sp, Se remained problematic, with little gain in Se compared with that obtained with a single sample.

When culturing milk samples for either research or clinical uses, a trade-off exists between expenditure of resources on additional milk samples from each mammary gland and increasing the number of cows (glands) sampled. Given the limited gains in Se and Sp observed with triplicate sampling, the use of this procedure will only be justified, from an economic perspective, if the cost of collecting and culturing additional milk samples is very low relative to the cost of increasing the sample size used in the study.

\section{Random Effects Models}

Random effects models were used to account for samples in this study that were not independent units but were clustered. This lack of independence means that failing to account for clustering will result in underestimation of standard errors (Dohoo et al., 2009), and hence, CI that are too narrow. As expected, the CI from the random effects models were much wider than those for the unconditional estimates. The discrepancy was most pronounced for the single sample estimates, because these were based on 4 observations taken from a single mammary gland and would have been highly correlated.

Random effects models produce different point estimates of Se and Sp than do unconditional associations (Dohoo et al., 2009) because they weight observations differently; they assign relatively more weight to individual observations derived from groups with small numbers of observations and less weight to those from large groups. In addition, the process of converting "subject-specific" estimates from random effects models to "marginal estimates" (to be comparable to unconditional estimates) depends on how well the variance components at each of the clustering levels are estimated. Any overestimation of these variance estimates will bias the resulting marginal estimates toward the null. In this study, the estimates of Se and Sp from the random effects models were all lower than the unconditional estimates. There was no reason to prefer these weighted estimates over the unconditional ones, so for simplicity and consistency with other estimates in the literature (particularly those reported by Dohoo et al., 2011), the unconditional estimates are the focus of this paper. The pattern of effects arising from the different sample combinations was similar from the 2 estimation procedures, with one exception. When using the random effects estimates to compare triplicate sampling to a single sample, a loss in Se was observed with triplicate sampling (59.8 to $51.3 \%$ ) and a slightly smaller gain in Sp (86.6 to $91.7 \%$ ) than in the unconditional estimates.

\section{CONCLUSIONS}

The Se and Sp of various combinations of samples were evaluated using data from 7,076 milk samples 
collected from 667 individual mammary glands from 176 cows in 8 herds. Series interpretation of duplicate or consecutive samples resulted in the highest Sp but the lowest Se. Parallel interpretation of duplicate or consecutive samples resulted in the highest Se but the lowest Sp. Overall, triplicate samples provided the best combination of Se and Sp but, compared with a single sample, provided only a modest gain in Sp and little or no gain in Se. In many situations, it may be difficult to justify the additional expense of triplicate sampling compared with single sampling. The selection of sampling method needs to be tailored to the goals of the study or clinical investigation.

\section{ACKNOWLEDGMENTS}

The study was funded by unrestricted grants from Minnesota DHI (Buffalo, MN) and Pfizer Animal Health (Madison, NJ). The graduate student (S. Andersen) was funded through the Canadian Bovine Mastitis Research Network (University of Montreal, St. Hyacinthe, Quebec, Canada). The authors thank Anna Bashiri (Ontario Veterinary College, Guelph, ON, Canada) and Russ Bey (University of Minnesota, St. Paul) for technical assistance as well as Jackie Conklin (Cornell University, Ithaca, NY) and Teresa Andrews (Atlantic Veterinary College, Charlottetown, PEI, Canada) for coordinating sample collection in New York and PEI, respectively.

\section{REFERENCES}

Andersen, S., I. R. Dohoo, R. Olde Riekerink, H. Stryhn, and Mastitis Research Workers' Conference. 2010. Diagnosing intramammary infections: Evaluating expert opinions on the definition of intramammary infection using conjoint analysis. J. Dairy Sci. 93:2966-2975.

Barkema, H. W., Y. H. Schukken, T. J. G. M. Lam, D. T. Galligan, M. L. Beiboer, and A. Brand. 1997. Estimation of interdependence among quarters of the bovine udder with subclinical mastitis and implications for analysis. J. Dairy Sci. 80:1592-1599.

Berry, D. P., and W. J. Meaney. 2006. Interdependence and distribution of subclinical mastitis and intramammary infection among udder quarters in dairy cattle. Prev. Vet. Med. 75:81-91.
Deluyker, H. A., S. N. v. Oye, and J. F. Boucher. 2005. Factors affecting cure and somatic cell count after pirlimycin treatment of subclinical mastitis in lactating cows. J. Dairy Sci. 88:604-614.

Djabri, B., N. Bareille, F. Beaudeau, and H. Seegers. 2002. Quarter milk somatic cell count in infected dairy cows: A meta-analysis. Vet. Res. 33:335-357.

Dohoo, I. R., S. W. Martin, and H. Stryhn. 2009. Veterinary Epidemiologic Research. 2nd ed. VER Inc., Charlottetown, PEI, Canada.

Dohoo, I. R., J. Smith, S. Andersen, D. F. Kelton, and S. Godden. 2011. Diagnosing intramammary infections: Evaluation of definitions based on a single milk sample. J. Dairy Sci. 94:250-261.

Erskine, R. J., and R. J. Eberhart. 1988. Comparison of duplicate and single quarter milk samples for the identification of intramammary infections. J. Dairy Sci. 71:854-856.

Halasa, T., K. Huijps, O. Osteras, and H. Hogeveen. 2007. Economic effects of bovine mastitis and mastitis management: A review. Vet. Q. 29:18-31.

International Dairy Federation. 1987. Bovine Mastitis Definition and Guidelines for Diagnosis. Vol. 211. IDF, Brussels, Belgium.

Jasper, D. E., J. D. Dellinger, and R. R. Bushnell. 1974. Agreement of duplicate samples of milk for the evaluation of quarter infection. Am. J. Vet. Res. 35:1371-1373.

National Mastitis Council. 1987. Laboratory and Field Handbook on Bovine Mastitis. National Mastitis Council, Arlington, VA.

National Mastitis Council. 1999. Laboratory and Field Handbook on Bovine Mastitis. Rev. Ed. National Mastitis Council, Verona, WI.

National Mastitis Council. 2004. Microbiological Procedures for Use in the Diagnosis of Bovine Udder Infection and Determination of Milk Quality. 4th ed. National Mastitis Council, Verona, WI.

Postle, D. S. 1976. Observations on bacteriologic isolation from pairs of quarter-milk samples. J. Am. Vet. Med. Assoc. 168:220-222.

Reyher, K. K., and I. R. Dohoo. 2011. Diagnosing intramammary infections: Evaluation of composite milk samples to detect intramammary infections. J. Dairy Sci. 94:3387-3396.

Seegers, H., C. Fourichon, and F. Beaudeau. 2003. Production effects related to mastitis and mastitis economics in dairy cattle herds. Vet. Res. 34:475-491.

StataCorp. 2009. Stata Statistical Software. Release 11. StataCorp LP, College Station, TX

Torres, A. H., P. J. Rajala-Schultz, and F. J. DeGraves. 2009. Diagnosis of intramammary infections at dry-off based on sampling strategy, epidemiology of pathogens, and agreement beyond chance. J. Vet. Diagn. Invest. 21:427-436.

US FDA. 1996. Revised April 4, 1996: Target animal safety and drug effectiveness studies for anti-microbial bovine mastitis products (lactating and non-lactating cow products). FDA Guideline. CVM GFI \#49: Animal Safety Study-Glossary of Terms. Public Health Service, US Department of Health and Human Services, Food and Drug Administration, Center for Veterinary Medicine, Washington, DC. 